\title{
Quantum massive conformal gravity
}

\author{
F. F. Faria ${ }^{a}$ \\ Centro de Ciências da Natureza, Universidade Estadual do Piauí, Teresina, PI 64002-150, Brazil
}

Received: 6 December 2015 / Accepted: 29 March 2016 / Published online: 7 April 2016

(C) The Author(s) 2016. This article is published with open access at Springerlink.com

\begin{abstract}
We first find the linear approximation of the second plus fourth order derivative massive conformal gravity action. Then we reduce the linearized action to separated second order derivative terms, which allows us to quantize the theory by using the standard first order canonical quantization method. It is shown that quantum massive conformal gravity is renormalizable but has ghost states. A possible decoupling of these ghost states at high energies is discussed.
\end{abstract}

\section{Introduction}

Massive conformal gravity [1] is a recently developed conformal theory of gravity in which the gravitational action is the sum of the fourth order derivative Weyl action [2] with the second order derivative Einstein-Hilbert action conformally coupled to a scalar field [3].

The gravitational potential of the theory, which is composed by an attractive Newtonian potential and a repulsive Yukawa potential, reproduces the rotation curves of the major number of galaxies. In addition, the momentum space propagators of massive conformal gravity have a good high-energy behavior, which makes the theory power-counting renormalizable. However, one of these propagators has a negative sign between its terms, which is a common feature of fourth order derivative theories of gravity. In such theories this negative sign imply that either the energy eigenvalue spectrum is unbounded from below or the Hilbert space norms are negative [4]. Several attempts to solve the negative energy (or negative norm) problem in higher derivative gravity have been carried out in the literature (see, e.g., [5-10]).

In this paper, we analyze the consequences of the negative sign term in massive conformal gravity. In Sect. 2 we derive a second order derivative linearized massive conformal gravity action by introducing auxiliary variables. In Sect. 3 we canonically quantize the massive conformal gravity fields and show

a e-mails: felfrafar@hotmail.com; fff@uespi.br that the theory has ghosts, states with negative norm, which do not necessarily spoil the unitarity of the $S$-matrix. Finally, in Sect. 4 we present our conclusions.

\section{Linearized action}

Let us consider the gravitational action of massive conformal gravity, which is given by ${ }^{1}$

$$
\begin{aligned}
S= & \frac{1}{2 k c} \int \mathrm{d}^{4} x \sqrt{-g}\left[\alpha\left(\varphi^{2} R+6 \partial_{\mu} \varphi \partial^{\mu} \varphi\right)\right. \\
& \left.-\lambda^{2} C^{\alpha \beta \mu \nu} C_{\alpha \beta \mu \nu}\right],
\end{aligned}
$$

where $\alpha$ is a dimensionless constants, $\lambda=\hbar / m c$ ( $\hbar$ is the Planck constant and $m$ is the graviton mass), $k=8 \pi G / c^{4}$ ( $G$ is the gravitational constant and $c$ is the speed of light in vacuum),

$$
\begin{aligned}
C_{\mu \beta \nu}^{\alpha}= & R_{\mu \beta \nu}^{\alpha}+\frac{1}{2}\left(\delta_{\nu}^{\alpha} R_{\mu \beta}-\delta_{\beta}^{\alpha} R_{\mu \nu}+g_{\mu \beta} R_{\nu}^{\alpha}\right. \\
& \left.-g_{\mu \nu} R^{\alpha}{ }_{\beta}\right)+\frac{1}{6}\left(\delta_{\beta}^{\alpha} g_{\mu \nu}-\delta^{\alpha}{ }_{\nu} g_{\mu \beta}\right) R
\end{aligned}
$$

is the Weyl tensor, $\varphi$ is a scalar field, $R^{\alpha}{ }_{\mu \beta \nu}$ is the Riemann tensor, $R_{\mu \nu}=R_{\mu \alpha \nu}^{\alpha}$ is the Ricci tensor, and $R=g^{\mu \nu} R_{\mu \nu}$ is the scalar curvature. It is worth noting that (1) is invariant under the conformal transformations

$\tilde{g}_{\mu \nu}=e^{2 \theta(x)} g_{\mu \nu}$,

$\tilde{\varphi}=e^{-\theta(x)} \varphi$,

where $\theta(x)$ is an arbitrary function of the spacetime coordinates.

\footnotetext{
$\overline{1}$ This action is equivalent to the action of Ref. [1]. The two actions have the same dimensions $\mathrm{Kg} \mathrm{m}^{2} / \mathrm{s}$. The only difference is that the action of Ref. [1] must have the mass measured in $\mathrm{Kg} \mathrm{m}^{2}$.
} 
With the help of the Lanczos identity, we can write (1) in the form

$$
\begin{aligned}
S= & \frac{1}{2 k c} \int \mathrm{d}^{4} x \sqrt{-g}\left[\alpha\left(\varphi^{2} R+6 \partial_{\mu} \varphi \partial^{\mu} \varphi\right)\right. \\
& \left.-2 \lambda^{2}\left(R^{\mu \nu} R_{\mu \nu}-\frac{1}{3} R^{2}\right)\right] .
\end{aligned}
$$

Then, using the weak-field approximations

$$
\begin{aligned}
& g_{\mu \nu}=g_{\mu \nu}^{(0)}+h_{\mu \nu}=\eta_{\mu \nu}+h_{\mu \nu}, \\
& \varphi=\varphi^{(0)}(1+\sigma)=\sqrt{\frac{2}{\alpha}}(1+\sigma),
\end{aligned}
$$

and keeping only the terms of second order in $h^{\mu \nu}$ and $\sigma$, we find that (5) reduces to

$$
\begin{aligned}
S= & \frac{1}{k c} \int \mathrm{d}^{4} x\left[\left(\overline{\mathcal{L}}_{E H}+2 \sigma \bar{R}+6 \partial_{\mu} \sigma \partial^{\mu} \sigma\right)\right. \\
& \left.-\lambda^{2}\left(\bar{R}^{\mu \nu} \bar{R}_{\mu \nu}-\frac{1}{3} \bar{R}^{2}\right)\right],
\end{aligned}
$$

where

$\bar{R}_{\mu \nu}=\frac{1}{2}\left(\partial_{\mu} \partial^{\rho} h_{\rho \nu}+\partial_{\nu} \partial^{\rho} h_{\rho \mu}-\partial_{\rho} \partial^{\rho} h_{\mu \nu}-\partial_{\mu} \partial_{\nu} h\right)$

is the linearized Ricci tensor,

$\bar{R}=\partial^{\mu} \partial^{\nu} h_{\mu \nu}-\partial_{\mu} \partial^{\mu} h$

is the linearized scalar curvature, and

$$
\begin{aligned}
\overline{\mathcal{L}}_{E H}= & -\frac{1}{4}\left(\partial^{\rho} h^{\mu \nu} \partial_{\rho} h_{\mu \nu}-2 \partial^{\mu} h^{\nu \rho} \partial_{\rho} h_{\mu \nu}+2 \partial^{\mu} h_{\mu \nu} \partial^{v} h\right. \\
& \left.-\partial^{\mu} h \partial_{\mu} h\right)
\end{aligned}
$$

is the linearized Einstein-Hilbert Lagrangian density, with $h=\eta^{\mu \nu} h_{\mu \nu}$.

The linearized action (8) is invariant under the coordinate gauge transformation

$h_{\mu \nu} \rightarrow h_{\mu \nu}+\partial_{\mu} \xi_{\nu}+\partial_{\nu} \xi_{\mu}$

where $\xi^{\mu}$ is an arbitrary spacetime dependent vector field, and under the conformal gauge transformations

$h_{\mu \nu} \rightarrow h_{\mu \nu}+\eta_{\mu \nu} \Lambda$

$\sigma \rightarrow \sigma-\frac{1}{2} \Lambda$

where $\Lambda$ is an arbitrary spacetime dependent scalar field. We can fix these gauge freedoms by imposing the coordinate gauge condition

$\partial^{\mu} h_{\mu \nu}-\frac{1}{2} \partial_{\nu} h=0$ and the conformal gauge condition

$\bar{R}-6 \lambda^{-2} \sigma=0$

to (8). However, this procedure is not suitable for the quantum analysis of the theory, since it introduces complication in the definition of the canonical commutation relations.

Another procedure to eliminate the gauge freedoms of the theory consists on adding gauge fixing terms to the action such that the field equations obtained from the action plus the gauge fixing terms are the same as the gauge fixed field equations obtained from the action alone. Thus, by adding the gauge fixing terms ${ }^{2}$

$$
\begin{aligned}
& S_{G F 1}=-\frac{1}{2 k c} \int \mathrm{d}^{4} x\left(\partial^{\mu} h_{\mu \nu}-\frac{1}{2} \partial_{\nu} h\right)^{2}, \\
& S_{G F 2}=\frac{1}{6 k c} \int \mathrm{d}^{4} x\left(\lambda \bar{R}-6 \lambda^{-1} \sigma\right)^{2}
\end{aligned}
$$

to (8), and integrating by parts, we obtain the diagonalized action

$$
\begin{aligned}
S_{d}= & -\frac{1}{2 k c} \int \mathrm{d}^{4} x\left[\frac{1}{2} \Psi^{\mu \nu}\left(\lambda^{2} \square-1\right) \square \Psi_{\mu \nu}\right. \\
& \left.+12 \sigma\left(\square-\lambda^{-2}\right) \sigma\right],
\end{aligned}
$$

where $\square=\partial_{\rho} \partial^{\rho}$ and

$\Psi_{\mu \nu}=h_{\mu \nu}-\frac{1}{2} \eta_{\mu \nu} h$

In order to obtain a first order canonical form, we choose the method of the decomposition into oscillator variables [11] and write the action (19) as

$$
\begin{aligned}
S_{d}= & \frac{1}{2 k c} \int \mathrm{d}^{4} x\left[\frac{1}{2} \Psi^{\mu \nu} \square \Phi_{\mu \nu}+\frac{1}{8} \lambda^{-2} \Psi_{\mu \nu} \Psi^{\mu \nu}\right. \\
& -\frac{1}{4} \lambda^{-2} \Psi_{\mu \nu} \Phi^{\mu \nu}+\frac{1}{8} \lambda^{-2} \Phi_{\mu \nu} \Phi^{\mu \nu} \\
& \left.-12 \sigma\left(\square-\lambda^{-2}\right) \sigma\right] .
\end{aligned}
$$

Varying this action with respect to $\Phi_{\mu \nu}$ gives

$\Phi_{\mu \nu}=\Psi_{\mu \nu}-2 \lambda^{2} \square \Psi_{\mu \nu}$,

and with this the field equations obtained from action (21) are equivalent to the field equations obtained from action (19). Finally, with the change of variables

$\Psi_{\mu \nu}=A_{\mu \nu}+B_{\mu \nu}$,

$\Phi_{\mu \nu}=A_{\mu \nu}-B_{\mu \nu}$

${ }^{2}$ In order to simplify the calculations we consider the Feynman gauge in which the Lagrange multipliers entering in the gauge fixing terms are equal one. 
we find the action

$$
\begin{aligned}
S_{d}= & \frac{1}{2 k c} \int \mathrm{d}^{4} x\left[\frac{1}{2} A^{\mu \nu} \square A_{\mu \nu}-\frac{1}{2} B^{\mu \nu}\left(\square-\lambda^{-2}\right) B_{\mu \nu}\right. \\
& \left.-12 \sigma\left(\square-\lambda^{-2}\right) \sigma\right],
\end{aligned}
$$

which is dynamically equivalent to action (8).

The action (25) contains a positive energy massless spin-2 field $A_{\mu \nu}$, a negative energy massive spin- 2 field $B_{\mu \nu}$, and a negative energy massive spin- 0 field $\sigma$. Classicaly, since the theory is not interacting, there is no problem with the negative energy fields. However, when interactions are introduced instabilities can appear. Thus a careful analysis is necessary on the interaction of massive conformal gravity with matter fields, which is beyond the scope of this paper. We will deal with the negative energy problem at the quantum level in the next section.

\section{Canonical quantization}

Varying the action (25) with respect to $A^{\mu \nu}, B^{\mu \nu}$, and $\sigma$, we obtain the field equations ${ }^{3}$

$$
\begin{aligned}
& \square A_{\mu \nu}=0, \\
& \left(\square-m^{2}\right) B_{\mu \nu}=0, \\
& \left(\square-m^{2}\right) \sigma=0 .
\end{aligned}
$$

The most general real solutions of these equations are given by

$$
\begin{aligned}
& A_{\mu \nu}(x)=\int \frac{\mathrm{d}^{3} p}{(2 \pi)^{3}} \frac{1}{\sqrt{2 \omega_{\mathbf{p}}^{A}}} \sum_{r}\left[a_{\mathbf{p}}^{r} \epsilon_{\mu \nu}^{r}(\mathbf{p}) e^{i p \cdot x}+c . c .\right] \\
& B_{\mu \nu}(x)=\int \frac{\mathrm{d}^{3} p}{(2 \pi)^{3}} \frac{1}{\sqrt{2 \omega_{\mathbf{p}}^{B}}} \sum_{s}\left[b_{\mathbf{p}}^{s} \varepsilon_{\mu \nu}^{s}(\mathbf{p}) e^{i p \cdot x}+c . c .\right], \\
& \sigma(x)=\int \frac{\mathrm{d}^{3} p}{(2 \pi)^{3}} \frac{1}{\sqrt{2 \omega_{\mathbf{p}}^{\sigma}}}\left[c_{\mathbf{p}} e^{i p \cdot x}+c . c .\right],
\end{aligned}
$$

where $\omega_{\mathbf{p}}^{A}=|\mathbf{p}|, \omega_{\mathbf{p}}^{B}=\sqrt{|\mathbf{p}|^{2}+m^{2}}, \omega_{\mathbf{p}}^{\sigma}=\sqrt{|\mathbf{p}|^{2}+m^{2}}$, the creation and annihilation operators obey the commutation relations

$\left[a_{\mathbf{p}}^{r}, a_{\mathbf{p}^{\prime}}^{r^{\prime} \dagger}\right]=(2 \pi)^{3} \delta^{3}\left(\mathbf{p}-\mathbf{p}^{\prime}\right) \delta^{r r^{\prime}}$,

$\left[b_{\mathbf{p}}^{s}, b_{\mathbf{p}^{\prime}}^{s^{\prime} \dagger}\right]=-(2 \pi)^{3} \delta^{3}\left(\mathbf{p}-\mathbf{p}^{\prime}\right) \delta^{s s^{\prime}}$,

$\left[c_{\mathbf{p}}, c_{\mathbf{p}^{\prime}}^{\dagger}\right]=-\frac{(2 \pi)^{3}}{24} \delta^{3}\left(\mathbf{p}-\mathbf{p}^{\prime}\right)$

with all the other commutators equal to zero, and the polarization tensors satisfy the orthonormality and completeness relations

\footnotetext{
$\overline{3}$ In this section we use "absolute units" in which $c=\hbar=16 \pi G=1$.
}

$\epsilon_{\mu \nu}^{r} \epsilon^{\mu \nu r^{\prime}}=\delta^{r r^{\prime}}$

$\varepsilon_{\mu \nu}^{s} \varepsilon^{\mu \nu s^{\prime}}=\delta^{s s^{\prime}}$

$\sum_{r} \epsilon_{\mu \nu}^{r} \epsilon_{\alpha \beta}^{r}=\frac{1}{2}\left(\eta_{\mu \alpha} \eta_{\nu \beta}+\eta_{\mu \beta} \eta_{\nu \alpha}-\eta_{\mu \nu} \eta_{\alpha \beta}\right)$,

$\sum_{s} \varepsilon_{\mu \nu}^{s} \varepsilon_{\alpha \beta}^{s}=\frac{1}{2}\left(\eta_{\mu \alpha} \eta_{\nu \beta}+\eta_{\mu \beta} \eta_{\nu \alpha}-\eta_{\mu \nu} \eta_{\alpha \beta}\right)$.

We can write (25) as

$S_{d}=\int \mathrm{d}^{4} x \mathcal{L}$

where

$$
\begin{aligned}
\mathcal{L}= & \frac{1}{2} A^{\mu \nu} \square A_{\mu \nu}-\frac{1}{2} B^{\mu \nu}\left(\square-m^{2}\right) B_{\mu \nu} \\
& -12 \sigma\left(\square-m^{2}\right) \sigma
\end{aligned}
$$

is the massive conformal gravity Lagrangian density. Using this Lagrangian density, we find the canonical momenta

$$
\begin{aligned}
& \Pi_{\mu \nu}=\frac{\partial \mathcal{L}}{\partial \dot{A}^{\mu \nu}}=-\dot{A}_{\mu \nu}, \\
& \Theta_{\mu \nu}=\frac{\partial \mathcal{L}}{\partial \dot{B}^{\mu \nu}}=\dot{B}_{\mu \nu}, \\
& \pi=\frac{\partial \mathcal{L}}{\partial \dot{\sigma}}=24 \dot{\sigma},
\end{aligned}
$$

where the dot denotes the time derivative.

It follows from (29)-(31) and (41)-(43) that

$$
\begin{aligned}
& \Pi_{\mu \nu}(x)=-\int \frac{\mathrm{d}^{3} p}{(2 \pi)^{3}} i \sqrt{\frac{\omega_{\mathbf{p}}^{A}}{2}} \sum_{r}\left[a_{\mathbf{p}}^{r} \epsilon_{\mu \nu}^{r}(\mathbf{p}) e^{i p \cdot x}+c . c .\right], \\
& \Theta_{\mu \nu}(x)=\int \frac{\mathrm{d}^{3} p}{(2 \pi)^{3}} i \sqrt{\frac{\omega_{\mathbf{p}}^{B}}{2}} \sum_{s}\left[b_{\mathbf{p}}^{s} \varepsilon_{\mu \nu}^{s}(\mathbf{p}) e^{i p \cdot x}+c . c .\right]
\end{aligned}
$$

$\pi(x)=24 \int \frac{\mathrm{d}^{3} p}{(2 \pi)^{3}} i \sqrt{\frac{\omega_{\mathbf{p}}^{\sigma}}{2}}\left[c_{\mathbf{p}} e^{i p \cdot x}+c . c.\right]$.

Thus, by imposing the commutation rules (32)-(34), we find

$$
\begin{aligned}
{\left[A_{\mu \nu}(x), \Pi_{\alpha \beta}(y)\right]=} & \frac{i}{2}\left(\eta_{\mu \alpha} \eta_{\nu \beta}+\eta_{\mu \beta} \eta_{\nu \alpha}-\eta_{\mu \nu} \eta_{\alpha \beta}\right) \\
& \times \delta^{3}(\mathbf{x}-\mathbf{y})
\end{aligned}
$$

$\begin{aligned} {\left[B_{\mu \nu}(x), \Theta_{\alpha \beta}(y)\right]=} & \frac{i}{2}\left(\eta_{\mu \alpha} \eta_{\nu \beta}+\eta_{\mu \beta} \eta_{\nu \alpha}-\eta_{\mu \nu} \eta_{\alpha \beta}\right) \\ & \times \delta^{3}(\mathbf{x}-\mathbf{y}),\end{aligned}$

$[\sigma(x), \pi(y)]=i \delta^{3}(\mathbf{x}-\mathbf{y})$,

with all the other commutators equal to zero. 
In order to find the energy spectrum of massive conformal gravity, we need the Hamiltonian of the theory, which is given by

$H=\int \mathrm{d}^{3} x \mathcal{H}$,

where

$\mathcal{H}=\Pi_{\mu \nu} \dot{A}^{\mu \nu}+\Theta_{\mu \nu} \dot{B}^{\mu \nu}+\pi \dot{\sigma}-\mathcal{L}$

is the massive conformal gravity Hamiltonian density. Substituting the Lagrangian density (40) and the canonical momenta (41)-(43) into (51), we arrive at

$$
\begin{aligned}
\mathcal{H}= & \frac{1}{2}\left(-\Pi_{\mu \nu} \Pi^{\mu \nu}+\partial_{i} A_{\mu \nu} \partial^{i} A^{\mu \nu}\right)+\frac{1}{2}\left(\Theta_{\mu \nu} \Theta^{\mu \nu}\right. \\
& \left.-\partial_{i} B_{\mu \nu} \partial^{i} B^{\mu \nu}-m^{2} B_{\mu \nu} B^{\mu \nu}\right)+\frac{1}{2}\left(\frac{1}{24} \pi^{2}\right. \\
& \left.-24 \partial_{i} \sigma \partial^{i} \sigma-24 m^{2} \sigma^{2}\right),
\end{aligned}
$$

where $\partial_{i}$ denotes the spatial derivatives, with $i=1,2,3$. Finally, after some calculation, we obtain

$$
\begin{aligned}
H= & \int \frac{\mathrm{d}^{3} p}{(2 \pi)^{3}} \sum_{r}\left(\omega_{\mathbf{p}}^{A} a_{\mathbf{p}}^{r \dagger} a_{\mathbf{p}}^{r}\right)-\int \frac{\mathrm{d}^{3} p}{(2 \pi)^{3}} \sum_{s}\left(\omega_{\mathbf{p}}^{B} b_{\mathbf{p}}^{s \dagger} b_{\mathbf{p}}^{s}\right) \\
& -\int \frac{\mathrm{d}^{3} p}{(2 \pi)^{3}}\left(\omega_{\mathbf{p}}^{\sigma} c_{\mathbf{p}}^{\dagger} c_{\mathbf{p}}\right),
\end{aligned}
$$

where we have dropped the infinite constants terms that comes from $\left[a_{\mathbf{p}}^{r}, a_{\mathbf{p}}^{r \dagger}\right],\left[b_{\mathbf{p}}^{s}, b_{\mathbf{p}}^{s \dagger}\right]$, and $\left[c_{\mathbf{p}}, c_{\mathbf{p}}^{\dagger}\right]$.

By imposing the commutation rules (32)-(34), and using the Hamiltonian (53), we find

$$
\begin{array}{ll}
{\left[a_{\mathbf{p}}^{r}, H\right]=\omega_{\mathbf{p}}^{A} a_{\mathbf{p}}^{r},} & {\left[a_{\mathbf{p}}^{r \dagger}, H\right]=-\omega_{\mathbf{p}}^{A} a_{\mathbf{p}}^{r},} \\
{\left[b_{\mathbf{p}}^{s}, H\right]=\omega_{\mathbf{p}}^{B} b_{\mathbf{p}}^{s}, \quad\left[b_{\mathbf{p}}^{s \dagger}, H\right]=-\omega_{\mathbf{p}}^{B} b_{\mathbf{p}}^{s},} \\
{\left[c_{\mathbf{p}}, H\right]=\omega_{\mathbf{p}}^{\sigma} c_{\mathbf{p}}, \quad\left[c_{\mathbf{p}}^{\dagger}, H\right]=-\omega_{\mathbf{p}}^{\sigma} c_{\mathbf{p}} .}
\end{array}
$$

If we choose the vacuum state $|0\rangle$ such that

$a_{\mathbf{p}}^{r}|0\rangle=0, \quad b_{\mathbf{p}}^{s}|0\rangle=0, \quad c_{\mathbf{p}}|0\rangle=0$,

then, using (54)-(56), we can show that the massless spin2 state $a_{\mathbf{p}}^{r^{\dagger}}|0\rangle$ is an eigenstate of $H$ with energy $\omega_{\mathbf{p}}^{A}$, the massive spin-2 state $b_{\mathbf{p}}^{s \dagger}|0\rangle$ is an eigenstate of $H$ with energy $\omega_{\mathbf{p}}^{B}$ and the massive spin-0 state $c_{\mathbf{p}}^{\dagger}|0\rangle$ is an eigenstate of $H$ with energy $\omega_{\mathbf{p}}^{\sigma}$. In this case, the energy spectrum of massive conformal gravity is bounded from below.

The Feynman propagators for the fields $A_{\mu \nu}, B_{\mu \nu}$ and $\sigma$ are, respectively,

$$
\begin{aligned}
D_{A}^{\mu \nu, \alpha \beta}(x-y)= & \left\langle 0\left|T\left(A^{\mu \nu}(x) A^{\alpha \beta}(y)\right)\right| 0\right\rangle \\
= & -\frac{i}{2}\left(\eta^{\mu \alpha} \eta^{\nu \beta}+\eta^{\mu \beta} \eta^{\nu \alpha}-\eta^{\mu \nu} \eta^{\alpha \beta}\right) \\
& \times \int \frac{\mathrm{d}^{4} p}{(2 \pi)^{4}} \frac{e^{-i p \cdot(x-y)}}{p^{2}-i \chi},
\end{aligned}
$$

$$
\begin{aligned}
D_{B}^{\mu \nu, \alpha \beta}(x-y)= & \left\langle 0\left|T\left(B^{\mu \nu}(x) B^{\alpha \beta}(y)\right)\right| 0\right\rangle \\
= & \frac{i}{2}\left(\eta^{\mu \alpha} \eta^{\nu \beta}+\eta^{\mu \beta} \eta^{\nu \alpha}-\eta^{\mu \nu} \eta^{\alpha \beta}\right) \\
& \times \int \frac{\mathrm{d}^{4} p}{(2 \pi)^{4}} \frac{e^{-i p \cdot(x-y)}}{p^{2}+m^{2}-i \chi},
\end{aligned}
$$

$$
\begin{aligned}
D_{\sigma}(x-y) & =\langle 0|T(\sigma(x) \sigma(y))| 0\rangle \\
& =i \int \frac{\mathrm{d}^{4} p}{(2 \pi)^{4}} \frac{e^{-i p \cdot(x-y)}}{p^{2}+m^{2}-i \chi},
\end{aligned}
$$

where $T$ denotes the time-ordered product and $\chi$ is an infinitesimal parameter. It follows from (23), (58), and (59) that the propagator for the field $\Psi_{\mu \nu}$ is

$$
\begin{aligned}
D_{\Psi}^{\mu \nu, \alpha \beta}= & -\frac{i}{2}\left(\eta^{\mu \alpha} \eta^{\nu \beta}+\eta^{\mu \beta} \eta^{\nu \alpha}-\eta^{\mu \nu} \eta^{\alpha \beta}\right) \\
& \times \int \frac{\mathrm{d}^{4} p}{(2 \pi)^{4}} \frac{m^{2} e^{-i p \cdot\left(x-x^{\prime}\right)}}{\left(p^{2}-i \chi\right)\left(p^{2}+m^{2}-i \chi\right)},
\end{aligned}
$$

which have a good $p^{-4}$ behavior at high momenta, making massive conformal gravity power-counting renormalizable.

The choice (57) gives the required positive energy spectrum and renormalization but produces difficulties in the normalization of the massive states. Using (32)-(34) and (57), we find the norms

$$
\begin{aligned}
& \left\langle 0\left|a_{\mathbf{p}}^{r} a_{\mathbf{p}^{\prime}}^{r^{\prime} \dagger}\right| 0\right\rangle=(2 \pi)^{3} \delta^{3}\left(\mathbf{p}-\mathbf{p}^{\prime}\right) \delta^{r r^{\prime}}, \\
& \left\langle 0\left|b_{\mathbf{p}}^{s} b_{\mathbf{p}^{\prime}}^{s^{\prime} \dagger}\right| 0\right\rangle=-(2 \pi)^{3} \delta^{3}\left(\mathbf{p}-\mathbf{p}^{\prime}\right) \delta^{s s^{\prime}}, \\
& \left\langle 0\left|c_{\mathbf{p}} c_{\mathbf{p}^{\prime}}^{\dagger}\right| 0\right\rangle=-\frac{(2 \pi)^{3}}{24} \delta^{3}\left(\mathbf{p}-\mathbf{p}^{\prime}\right),
\end{aligned}
$$

and thus $b_{\mathbf{p}}^{s \dagger}|0\rangle$ and $c_{\mathbf{p}}^{\dagger}|0\rangle$ are ghost states, ${ }^{4}$ which renders the theory non-unitary. However, if the mass $m$ of both massive particles is greater than the available energy then it does not give rise to stable ghost states. In this case, the $S$-matrix connects only asymptotic states with positive norm and thus it is a unitary matrix. Since massive conformal gravity is renormalizable, we can apply the standard renormalization group methods to study the high-energy behavior of $m$, which is not a trivial thing to do. So we will leave these calculations for future work.

${ }^{4}$ Alternatively, if we choose $a_{\mathbf{p}}^{r}|0\rangle=0, b_{\mathbf{p}}^{s^{\dagger}}|0\rangle=0$, and $c_{\mathbf{p}}^{\dagger}|0\rangle=0$, then the theory is free of ghost states, but the energy spectrum is unbounded from below. 


\section{Final remarks}

We have shown in this paper that massive conformal gravity is a renormalizable quantum theory of gravity which has two massive ghost states. A careful analysis is needed to check if these ghost states are decoupled from the theory at high energies, which would ensure the unitarity of the theory. While this analysis is not done we cannot rule out massive conformal gravity as a viable quantum theory of gravity. We are investigating this issue right now and we hope that this investigation help to show that quantum massive conformal gravity is not only renormalizable but also unitary.

Open Access This article is distributed under the terms of the Creative Commons Attribution 4.0 International License (http://creativecomm ons.org/licenses/by/4.0/), which permits unrestricted use, distribution, and reproduction in any medium, provided you give appropriate credit to the original author(s) and the source, provide a link to the Creative Commons license, and indicate if changes were made.

Funded by SCOAP ${ }^{3}$.

\section{References}

1. F.F. Faria, Adv. High Energy Phys. 2014, 520259 (2014)

2. H. Weyl, Spacetime Matter (Dover, New York, 1952)

3. P.A.M. Dirac, Proc. R. Soc. Lond. A 333, 403 (1973)

4. K.S. Stelle, Phys. Rev. D 16, 953 (1977)

5. E. Tomboulis, Phys. Lett. B 70, 361 (1977)

6. A. Salam, J. Strathdee, Phys. Rev. D 18, 4480 (1978)

7. I. Antoniadis, E.T. Tomboulis, Phys. Rev. D 33, 2765 (1986)

8. S.W. Hawking, T. Hertog, Phys. Rev. D 65, 103515 (2002)

9. G. Narain, R. Anishetty, J. Phys. Conf. Ser. 405, 012024 (2012)

10. T. Biswas et al., Phys. Rev. Lett. 108, 031101 (2012)

11. A. Pais, G.E. Uhlenbeck, Phys. Rev. 79, 145 (1950) 